Bull. Mater. Sci., Vol. 22, No. 6, October 1999, pp. 1003-1008. (C) Indian Academy of Sciences.

\title{
Investigation of electrical conduction in polyvinyl formal
}

\author{
P K KHARE*, R K PANDEY and P L JAIN" \\ Department of Physics, Dr H S Gaur University, Sagar 470 001, India \\ "Government Postgraduate College, Damoh, India \\ MS received 16 March 1999; revised 28 July 1999
}

\begin{abstract}
Current-voltage ( $I-V$ ) characteristics of pure polyvinyl formal (PVF) were investigated at different fields, range $5-100 \mathrm{kV} / \mathrm{cm}$, as a function of temperature, range $313-363 \mathrm{~K}$. It was observed that while at low fields (up to $25 \mathrm{kV} / \mathrm{cm}$ ), the conduction was Ohm's law-dependent at high fields (beyond $25 \mathrm{kV} / \mathrm{cm}$ ), the conduction was Poole-Frenkel (P-F) mechanism-dependent. An attempt was made to identify the nature of the current by comparing its observed dependence on temperature, electric field and electrode materials with their respective characteristic features of the existing theories of electrical conduction. The current showed a strong dependence on temperature. To identify the possible mechanism of conduction, current versus square root of field characteristics were drawn with aluminium, silver, copper and gold as upper electrodes and Al as the lower electrodes. The observed characteristic suggested that the charge carriers were generated by the fieldassisted lowering of coulombic barriers at the traps, and were subsequently conducted through the bulk of the material by a hopping process between the localized states by a Jonscher-Ansari-modified P-F mechanism. The calculated value of the modified P-F barrier was $\simeq 1.94 \times 10^{-19} \mathrm{~J}(1.21 \mathrm{eV})$.
\end{abstract}

Keywords. Electrical conduction; polyvinyl formal; Poole-Frenkel mechanism.

\section{Introduction}

The last decade has seen an ever increasing interest in the electrical properties of polymers (Bahri and Singh 1980; Khare and Srivastava 1991; Keller et al 1993; Khare 1994; Khare et al 1994a; Khare and Chandok 1995). The polymers have industrial uses owing to their low production cost and ease of manufacture. To miniaturize the electrical equipments, the demand for organic polymers as electrical insulators, which are stable at high temperatures, is increasing. However, the exact mechanism of conduction of current in polymers is not understood due to: (i) presence of considerable amount of disordered material in them, (ii) their high molecular weight and (iii) their having some unique physical and chemical properties. Moreover, the electrical behaviour of the insulating materials on the other hand can also be attributed to the trapping centre, presence of pendent side groups, dipole chain folds, and the role of disorders. However, in most cases, the electrical behaviour of the insulating materials is controlled either by Schottky emission or the Poole-Frenkel (P-F) effect in the moderately high field region. But since both Schottky emission as well as $\mathrm{P}-\mathrm{F}$ exhibit similar $I-V$ relationship, sometimes it is difficult to decide as to which of the two mechanisms is the controlling mechanism. In addition,

\footnotetext{
*Author for correspondence
}

Jonscher and Ansari (1971) proposed a modified P-F model which accounted for the low mobility of the charge carriers as well. In this paper, we have presented the results of our investigations on the validity of the proposed identification method for the modified P-F model.

We have studied the effect of temperature and the applied field on the conductivity of solution-grown polyvinyl formal films (PVF) in order to understand the possibility of the existence and nature of distribution of traps, and the role played by carriers injected from the electrodes and volume-generated charge carriers. The studies on electronic transport phenomena in polyvinyl formal were carried out to understand the basic electronic transport mechanism in the macromolecular material so that we can improve the stability characteristics of the material, which are the key factors in governing device performance.

\section{Experimental}

Polyvinyl formal having a glass transition temperature $\approx 381 \mathrm{~K}$ and molecular weight $(50,000)$ was obtained from GLAXO Co., Bombay. For the preparation of the films, the polymer was used without further purification/ recrystallization. The isothermal immersion technique was utilized for preparing thin films of PVF. PVF has a nonpolar main chain with a weakly polar $\mathrm{CO}$ group 
rigidly attached to the main chain. The repeat unit of PVF is given below:<smiles>CCOCC1COCCO1</smiles>

The solution was prepared in a glass beaker by dissolving $4.2 \mathrm{~g}$ PVF in a solvent containing a mixture of benzene and methanol, ratio $3: 2$, at room temperature. For $30 \mathrm{~min}$, the solution was continuously stirred using a teflon-coated magnetic stirrer. Thereafter, it was stirred and heated to $313 \mathrm{~K}$ to yield a homogeneous solution. The glass beaker with the solution was next immersed in a constant temperature oil bath. For the next $30 \mathrm{~min}$, microscopic glass slides, which were ultrasonically cleaned, and vacuum-metallized were immersed vertically into the solution. After deposition of the film, the glass slides were taken out and dried in an oven at $313 \mathrm{~K}$ for $24 \mathrm{~h}$. This was followed by room temperature outgassing at $133.33 \times 10^{-5} \mathrm{~N} / \mathrm{m}^{2}$ for a further period of $24 \mathrm{~h}$. The film thickness was determined capacitively at $50 \mathrm{~Hz}$ on the basis of the known dielectric constant of PVF (Bandrup and Immergut 1975). Prior to the conduction measurement, the samples were heated with the electrodes shorted at a heating rate of $4^{\circ} \mathrm{C} / \mathrm{min}$ and held at $70^{\circ} \mathrm{C}$ for $20 \mathrm{~min}$ so as to minimize the data scatter from sample to sample. Thirty minutes after the attaining of the temperature $T_{p}$, a step field was applied to the film and the electric current was measured. The geometry of the sandwich configuration of the electrodes was the same as reported earlier (Khare et al 1993, 1994b). The voltage was applied from a high voltage unit (EC-HV $4800 \mathrm{D}$ ), and the steady state current was measured by means of an electrometer, Keithley $600 \mathrm{~B}$, which was carefully shielded and grounded so as to avoid ground loops or extraneous electrical noise.

\section{Results and discussion}

Figure 1 shows the current-voltage $(I-V)$ characteristics of PVF films at temperatures ranging from $313-363 \mathrm{~K}$. The observed increment in the current was approximately the same throughout the studied range of temperature. For all the temperatures, the thermograms, though nonlinear, were similar. A slope of 2 generally indicates shallow trapping, although the possibility for the presence of deep traps in the present case, can not be excluded. The $\ln I-V$ plots (figure 1) of all the samples showed the slope in the low-field region, up to $25 \mathrm{kV} / \mathrm{cm}$, as 1 . In contrast, in the high-field region, the value of slopes was greater than 1 , but not exceeding 2 . The slope value of 1 , in the low-field region, indicate that the electrical conductance in this region obeys Ohm's law. However, since the complete $I-V$ characteristics were nonlinear, they indicate the presence of other modes of electrical conduction as well. The observed nonlinearity could be due to build up of a space charge, resulting in nonuniformity of field distribution between the electrodes. The slope value for the field region $>25 \mathrm{kV} / \mathrm{cm}$ is $>1$ and $<2$, indicates that the electrical conduction in this region is not through space charge limited current (SCLC). Moreover, the linearity of $I-V$ curves in the high-field region indicates that either of the mechanisms, Schottky Richardson or Poole-Frenkel, may be operative.

On the basis of the various factors governing the variation of current with temperature, i.e. (i) nature of trap distribution in the sample, (ii) presence of impurity in the sample, and (iii) production of defects due to thermal excitation; O'Dwyer (1966) suggested presence of numerous isolated shallow traps covering an energy range

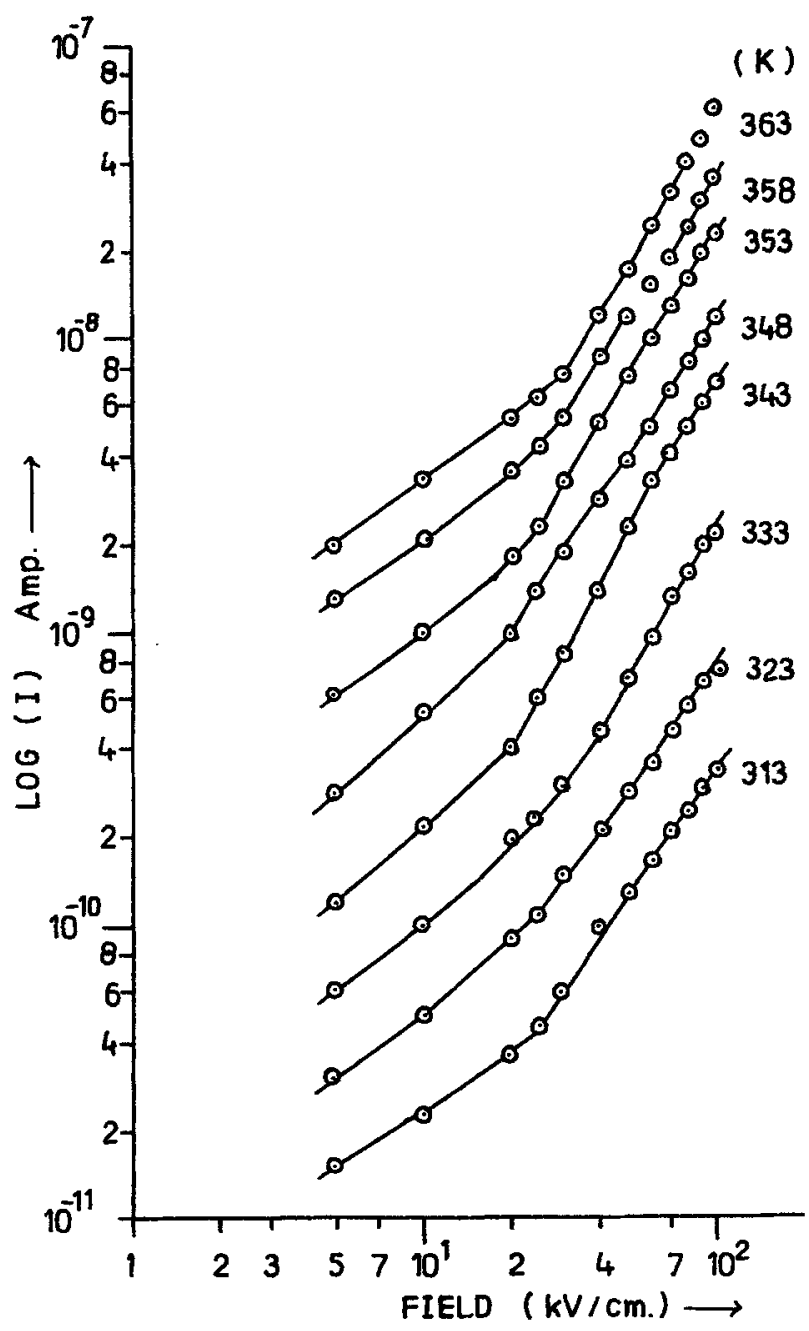

Figure 1. Plot of $\log I$ versus $\log V$ at various temperatures $(313,323,333,343,348,353,358$ and $363 \mathrm{~K})$. 
$\Delta W$ below the continuum of free electron levels. Presence of deep traps, whose energy $W$ is between the conduction and valency levels, is also a possibility. While at sufficient low temperature (absolute zero), all the trapped electrons would be in deep traps, at a finite temperature, in the presence of an applied field, some of these electrons can be excited into the shallower traps (or conduction levels), either thermally or due to action of the applied field. Under the influence of the applied field, these electrons participate in electrical conduction. The increase in temperature, however, does not alter the total amount of space charge in the conduction band, which increases exponentially as inverse of the absolute temperature.

To explain the mode of electron transport in thin insulating films, the various proposed mechanisms are: (i) tunnelling (Fischer and Giaver 1961), (ii) Schottky emission (Emlager and Tantaporn 1962), (iii) field and thermal ionization of traps and impurities (Mead 1962), (iv) avalanche multiplication (Advani et al 1962), (v) Poole-Frenkel emission (Myioshisi and Chino 1967), and (vi) space charge-limited conduction. In addition, many workers engaged in polymer research have proposed electronic (Fowler 1956; Bashara and Doty 1964), ionic (Roggern 1962; Reiser et al 1969) and protonic (Elay and Spivey 1961; Mann 1964) conduction for polymers.

Our results indicate that with increasing temperature, the probability of thermal ionization of the trapping centres also increases, resulting thereby in a shift in the quasi-Fermi level. This shift lowers the barrier across which electrons are transported, and the electrical conduction is ohmic. At low-energy fields, fewer carriers are injected from the electrodes, and therefore the initial current is governed by the intrinsic free carriers in the materials. At higher-energy fields, the change of slope is suggestive of deviation from Ohm's law-based conduction to the space charge-limited conduction. This mode of electrical conduction continues until the injected free carrier density becomes comparable with the thermally created carrier density. However, at sufficiently higherenergy fields, the current is dominated by space-charge limited conduction and the conduction is mainly due to injected space charge (Khare et al 1994c). The departure from Ohm's law in high-energy field is due to perturbation of active barrier even in the absence of thermal and chemical effects. Since, for. our studies, the film thickness was very high $(25 \mu \mathrm{m})$, the conduction mechanism by tunnelling is ruled out, which requires the film thickness to be very thin $(\leq 50 \AA)$.

The observed relation between the current and voltage points to a conduction mechanism in which the charge carriers are released by thermal activation over a coulombic potential barrier that decreases with the applied electric field. The physical nature of such a potential barrier can be interpreted in two basic ways: (i) barrier between the electrodes and dielectric taking the classic image force into consideration (Schottky emission), and (ii) barriers due to trapping centres in the dielectric-Poole-Frenkel (P-F), effect.

While the expression for the current density in Schottky emission is:

$$
J_{\mathrm{SR}}=A^{*} T^{2} \exp (-\phi / k T) \exp \left[e / k T(e E / 4 \pi \varepsilon)^{1 / 2}\right],
$$

where $J$ is the current density, $e$ the electronic charge, and $E$ the field applied at $T$ (in $k$ ) and $A^{*}$ and $\phi$ are the Richardson constant and potential barrier, respectively at the metal-insulator interface in the absence of a field, the current-voltage relationship in Poole-Frenkel (P-F) mechanism is given by:

$$
J_{\mathrm{P}-\mathrm{F}}=C E \exp (-\phi / 2 k T) \exp \left[e / k T(-e E / \pi \varepsilon)^{1 / 2}\right],
$$

where $C$ is the constant independent of field and temperature, and $\phi$ is the barrier height.

To determine the actual conduction mechanism, the value of $\beta$-factor, at different temperatures, calculated from the slope of the plots of $\log l$ versus $\sqrt{E}$ (figure 2), were compared with the theoretically calculated value of $\beta$, based on the premise that one of the mechanisms was operating. The experimental values of $\beta$ (table 1), in the present case, were close to the theoretically arrived values of $\beta_{\mathrm{P}-\mathrm{F}}$ based on the premise that P-F was the particular

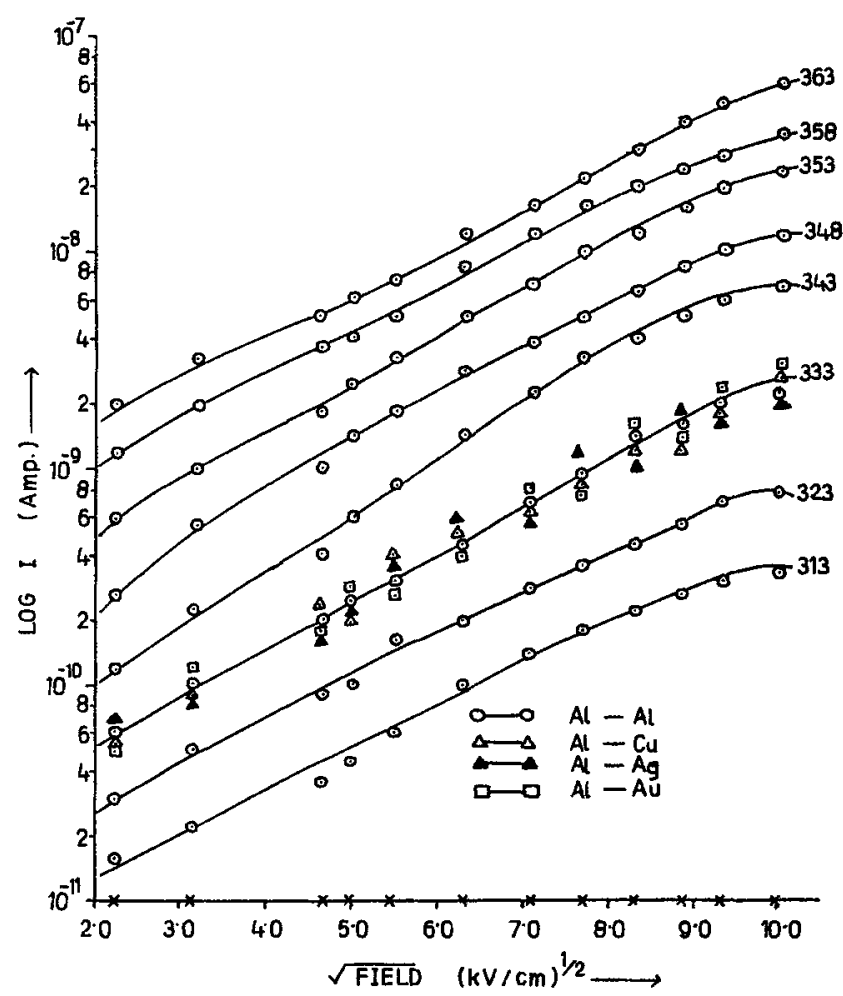

Figure 2. Plot of $\log I$. versus $\sqrt{ } E$ at various temperatures (313, $323,333,343,348,353,358$ and $363 \mathrm{~K}$ ) with similar and dissimilar electrode combination. 
mechanism that was operating. Jonscher and Ansari (1971) pointed out that instead of basing the distinction between the two mechanisms, on the magnitude of $\beta$, it should be based on studies taking an asymmetric $\mathrm{M}-\mathrm{I}-\mathrm{M}$ structure with two electrodes of different work functions. The current in the case of Schottky effect will be asymmetrical when polarities are reversed. On the other hand, it will remain practically unchanged in the case of $\mathrm{P}-\mathrm{F}$ effect, since it does not depend on potential barrier at the interfaces, i.e. the basic difference between them is: one is electrode-dependent and the other is not. This can be further substantiated by a study of thermally stimulated discharge currents as a function of polarizing field strength, to determine the actual conduction mechanism. Consequently, a graph of $\log I$ versus $\sqrt{ } E$ was plotted for different electrode materials (aluminium, silver, copper and gold) at $333 \mathrm{~K}$, the other electrode being of aluminium in each case (figure 2). Since a single straight line was obtained within the limits of experimental error, the presence of an electrode-limited RS mechanism can perhaps be ruled out.

But, in the present investigation, the P-F model of conduction, in its conventional form, cannot be applied. The conventional model assumes a field-assisted thermal excitation of electrons from traps situated in the conduction band of insulator. Once the electron has acquired sufficient energy for reaching the conduction band, it is free to move unhindered and unobstructed. But Mott and Davis (1971) pointed out that a drift mobility, less than about $1 \mathrm{~cm}^{2} \mathrm{~V}^{-1} \mathrm{~S}^{-1}$, cannot correspond to this unhindered free-hand conduction. Taking this into account, a modified model for conduction was therefore proposed by Jonscher and Ansari (1971) for substances of low mobility. This model involves the use of a large number of localized trap states, randomly distributed in space and energy. Mott and Davis (1971) too conjectured the presence of trap states in disordered structures. In polymers, these traps may be a result of regular chain folding, their (chain) termination, the presence of crystallineamorphous interfaces, chain entang lements etc.

The new model furthermore suggests that the process of field-assisted ionization of donor-like carriers, which is the basis of P-F mechanism, does not produce free carriers in a conduction band, as is normally considered to be the case, but merely causes hopping-like movements of localized carriers in the trap levels. This process of hopping is assisted by the presence of an external field which reduces 'hop barriers' by $v E^{1 / 2}$, where $v=\left(e^{3} / \pi \varepsilon \varepsilon_{0}\right)^{1 / 2}$, as given in the conventional $\mathrm{P}-\mathrm{F}$ model. The revised expression can thus be written as:

$$
I=I_{0} \exp \left[-\left(W_{0}-v E^{1 / 2}\right) / k T\right]
$$

where $W_{0}$ is the average barrier height of 'hops', $I_{0}$ the pre-exponential coefficient that may be defined as the current at an infinite temperature, $e$ the electronic charge, $\varepsilon$ the high-frequency dielectric constant and $\varepsilon_{0}$ the permittivity of space charge.

Figure 3 shows the Arrhenius plots between $\log I$ versus $10^{3} / T$ at different fields for polyvinyl formal samples. In the plot of figure 3 , two distinct regions with different slopes at lower and higher temperature can be seen, showing different associated thermal activation energies as well in the two regions. The low-temperature slope is an arbitrary one and depicts the complex temperature dependence of distributed relaxation processes. This break point corresponds to the glass transition temperature $\left(T_{\mathrm{g}}\right)$ of the polymer.

It is well established that the glass transition is a relaxation phenomenon that occurs over the temperature range wherein the relaxation time of an internal mechanism, resulting in increased motion and segmental mobility of the polymer, is of the order of time scale of the experiment. According to Turnhout (1975), conductivity in polymers show a sharp increase near $T_{\mathrm{g}}$. The dependence of the current on temperature shows two distinct regions, i.e. one below $T_{\mathrm{g}}$ and the other beyond $T_{\mathrm{g}}$. The conduction below $T_{\mathrm{g}}$ takes place by thermal activation of carriers from one disordered state to another. At $T_{g}$, molecular motion of the chain segments lowers the trap depth considerably and trapped charges are released by thermal excitation and above $T_{\mathrm{g}}$, the temperature is sufficiently high to cause segmental chain motion which mobilizes the traps; therefore the conductivity suddenly increases above $T_{\mathrm{g}}$. At low temperature, current is low. The small current is due to trapping of injected charge carriers from electrodes at different levels (Tripathi and Srivastava 1982). Detrapping from these levels require

Table 1. Theoretical and experimental values of $\beta$ and the average barrier height of hops $\left(W_{0}\right)$.

\begin{tabular}{ccccc}
\hline $\begin{array}{c}\text { Temp. } \\
(\mathrm{K})\end{array}$ & $\begin{array}{c}\text { Experimental } \\
\boldsymbol{\beta} \text {-values } \\
10^{-24} \mathrm{~J} \mathrm{M}^{1 / 2} \mathrm{~V}^{-1 / 2}\end{array}$ & $\begin{array}{c}\beta_{\mathrm{SR}}^{*} \\
10^{-24} \mathrm{~J} \mathrm{M}^{1 / 2} \mathrm{~V}^{-1 / 2}\end{array}$ & $\begin{array}{c}\beta_{\mathrm{P}}^{* *} \\
10^{-24} \mathrm{~J} \mathrm{M}^{1 / 2} \mathrm{~V}^{-1 / 2}\end{array}$ & $\begin{array}{c}W_{0} \text { (from figure 3) } \\
(\mathrm{eV})\end{array}$ \\
\hline 313 & 3.887 & 2.453 & 4.906 & \\
323 & 6.112 & 3.729 & 7.458 & 1.16 \\
333 & 6.324 & 3.992 & 7.984 & \\
348 & 6.992 & 4.254 & 8.508 & \\
363 & 7.132 & 4.332 & 8.664 & \\
\hline
\end{tabular}

* $\beta_{\mathrm{SR}}$ : Schottky-Richardson-based $\beta$ values.

$* * \beta_{\mathrm{P}-\mathrm{F}}$ : Poole-Frenkel-based $\beta$ values. 
increased molecular motion of polymer chains. When thermal energy is supplied to a polymer, its temperature increases which induces various transitions in them. Increased molecular motion due to increase of temperature results in thermal excitation of charge carriers from the traps, either directly or due to lowering of trap depth. Molecular motions also result in release of trapped carriers due to loss of trapping sites (Srivastava and Agrawal 1974).

Since the commercial PVF, we used for this study, was a mixture of amorphous and crystalline regions; the conductivity behaviour of solution-grown films of this polymer may be dominated by the properties of amorphous regions in the film, because many localized states are present in these regions. In fact, Brown and Aftergut (1963) considered the presence of two slopes as an indication of impurities, and the resulting current, in an impure semiconductor current being due to two competing conduction processes acting simultaneously: in the first process, an electron occupying an isolated donor has a wavefunction located about the impurity and an energy slightly below the conduction band minimum. Because there is a small, but finite, overlap of the wavefunction of an electron on one donor with those of the neighbouring donors, a conduction process becomes a possibility in cases where the electron moves between the centres, due to tunnelling effect, without its activation into the

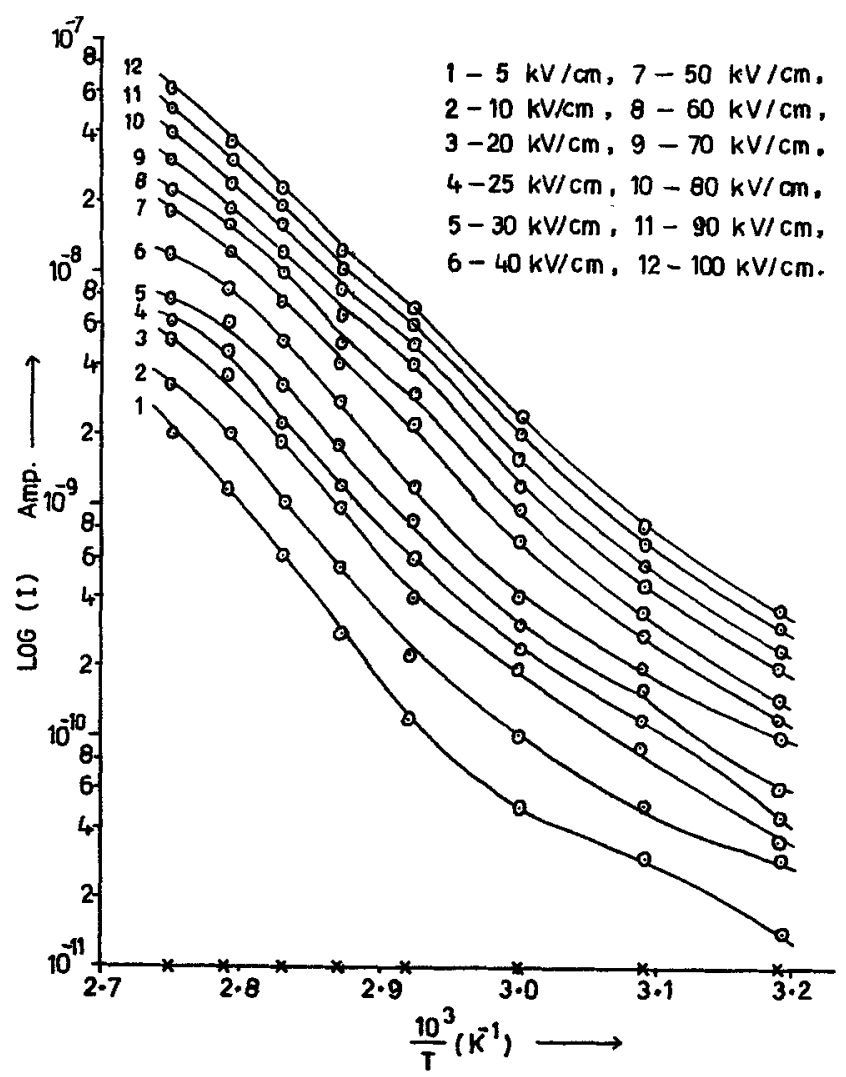

Figure 3. Plot of $\log I$ versus $10^{3} / T$ at different fields. conduction band; in the second process the current, which is carried by electrons in the conduction band, is usually observed to be in thermal equilibrium with electrons or donor impurities (Tripathi and Srivastava 1982).

The activation energy values for the various fields calculated from these plots were $0.30-0.78 \mathrm{eV}$. The low values of activation energy $(<1 \mathrm{eV})$ indicate the predominance of electronic conduction in the present case. The activation energy values decrease with the applied field, suggesting that the potential barriers are lowered due to the applied field. This suggests that the applied field interacts with the field of the ionizable centre so that the energy required for an electron to escape in a direction opposite to the field direction (i.e. in the field-assisted or 'forward' direction) is reduced by an amount.

The P-F mechanism assumes that once the carriers are thermally activated from their trapping sites, with the assistance of the field, they are free to move unobstructed within the conduction band of the insulator. According to Jonscher and Ansari (1971), conduction in polymeric dielectrics with low mobility charge carriers should however be interpreted as conduction by localized charge carriers performing hopping. The modified P-F mechanism predicts an activation energy which decreases with the square root of the applied field. Figure 4 shows the variation of observed activation energy with the square root of the applied field. The intercept of the obtained straight line at zero field while gives a value of $W_{0}$ of $1.94 \times 10^{-19} \mathrm{~J}(1.21 \mathrm{eV})$, its slope yields a value of $6.197 \times 10^{-24} \mathrm{~J} \mathrm{M}^{1 / 2} \mathrm{~V}^{-1 / 2}$, which is in fair agreement with

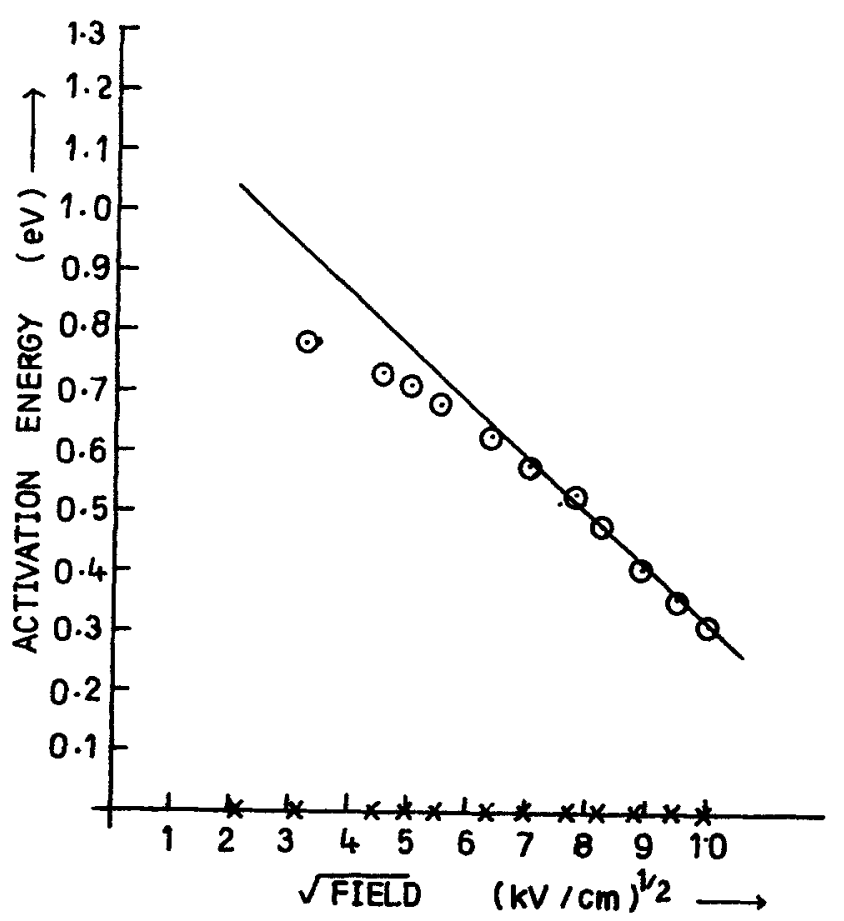

Figure 4. Plot of activation energy versus square root of the field. 
the theoretical value of $\beta_{\mathrm{PF}}\left(7.355 \times 10^{-24} \mathrm{~J} \mathrm{M}^{1 / 2} \mathrm{~V}^{-1 / 2}\right)$. This further confirms that the modified P-F mechanism is the operating conduction mechanism in the present case.

In the thermally stimulated depolarization current (TSDC) measurement, the observed peaks may be due to disorientation of dipoles or migration of space charge carriers over microscopic distances released from the traps, with subsequent trapping (Khare et al 1999). If the trapping centres are indeed of a Poole-Frenkel type, the temperature corresponding to the TSDC peak should be dependent on . the polarizing field strength. It was observed that the temperature corresponding to the peak was indeed a function of field strength.

\section{Conclusion}

The current-voltage data of the present study fits fairly well with the Poole-Frenkel mechanism of conduction modified by Jonscher and Ansari.

\section{References}

Advani G T, Gottling J S and Oswan M S 1962 Proc. IRE 50 1530

Bahri R and Singh H P 1980 Thin Solid Films 69281

Bandrup J and Immergut E H 1975 Polymer handbook (New York: John Wiley) Vol. VIII, p. 7

Bashara N M and Doty C T 1964 J. Appl. Phys. 353498

Brown G P and Aftergut S 1963 J. Chem. Phys. 381356

Christy M M 1965 J. Appl. Phys. 161805

Emlager P R and Tantaporn L 1962 Phys. Rev. Lett. 8262
Elay D D and Spivey D L 1961 Trans. Faraday Soc. 572280

Fischer J C and Giaver I 1961 J. Appl. Phys. 32172

Fowler J F 1956 Proc. R. Soc. (London): J. Appl. Phys. 236456

Jonscher A K and Ansari A A 1971 Philos. Mag. 23205

Keller J M, Datt S C, Dubey R, Singh R and Khare P 1993 Phys. Status Solidi 139391

Khare P K, Jain S K and Jain P L 1999 (To be published)

Khare P K and Chandok 1995 J. Polym. Mater. 1223

Khare P K 1994 Indian J. Pure \& Appl. Phys. 32160

Khare P K, Gaur M S and Srivastava 1994a Indian J. Pure \& Appl. Phys. 2314

Khare P K, Shrivastava Sandeep and Srivastava A P 1994b Indian J. Phys. 68129

Khare P K, Keller J M, Gaur M S, Singh Ranjeet and Datt S C 1994c Polym. Int. 35337

Khare P K, Gaur M S, Bajpai Alka, Pandey R K and Srivastava A P 1993 Indian J. Pure \& Appl. Phys. 31326

Khare Pawan and Srivastava A P 1991 Indian J. Pure \& Appl. Phys. 29410

Mann A T 1964 J. Appl. Phys. 352173

Mead C A 1962 Phys. Rev. 1282088

Myjoshisi Y and Chino K 1967 J. Appl. Phys. 6181

Mott N F and Davis E A 1971 Electronic processes in non crystalline materials (Oxford: Clarendon Press)

O'Dwyer J J 1966 J. Appl. Phys. 37599

Reiser A, Lock M W B and Enight J 1969 Trans. Faraday Soc. 652168

Roggern A Von 1962 Phys. Rev. Lett. 9368

Srivastava A P and Agrawal S R 1974 Phys, Lett. 322

Tripathi A K and Srivastava A P 1982 Indian J. Pure \& Appl. Phys. 20863

Turnhout J Van 1975 Thermally stimulated discharge of polymer electrets (New York) 